\title{
Misconceptions and facts about treating hypertension
}

\author{
Franz H. Messerli and Stefano F. Rimoldi
}

The overall prevalence of hypertension is high and likely to further increase with aging, obesity and other risk factors. In fact, in the United States, the International Statistical Classification of Diseases and Related Health Problems code for hypertension is by far the most common single code used by practicing physicians. Although the benefits of antihypertensive therapy have been established by a solid bulk of evidence and rates of hypertension awareness, treatment and control have been improving over recent decades; they are still far from adequate. Moreover, several misconceptions persist among practicing clinicians, commonly in the areas in which evidence is scarce, misinterpreted or substituted by eminence.

For instance, hydrochlorothiazide remains by far the most commonly prescribed antihypertensive agent in the United States and worldwide. Yet, as pointed out by Dr Wanpen Vongpatanasin (pp. 361365 ) in her provocative review, hydrochlorothiazide in its usual dose of 12.5-25 mg per day has never been shown to reduce the risk of MI, stroke or death. Also, meta-analysis of clinical trials showed that, for any given difference in achieved clinic SBP, hydrochlorothiazide therapy was associated with $18 \%$ higher adverse cardiovascular events when compared with chlorthalidone. Clearly, therefore, chlorthalidone or indapamide should be preferred over hydrochlorothiazide in hypertensive patients whenever diuretics are required for the treatment of hypertension.

Dr Steven G. Chrysant (pp. 383-390) tackles another common misconception, namely that antihypertensive therapy causes erectile dysfunction (ED). Indeed, erectile dysfunction is common in treated hypertensive men. However, as Dr Chrysant suggests, we have to ask whether the ED is due to hypertension per se, the antihypertensive regimen or a combination of both. Of note, data from randomized studies on ED and antihypertensive therapy are limited; as of today, the issue remains very much an evidence-free zone. It should not be forgotten that knowledge by the patient of the drug and its action on erectile dysfuction (ED) may increase the incidence of ED (Hawthorn's effect).

Dual renin-angiotensin blockade was very much in fashion in the early part of this century; primarily accepted and promoted by nephrologists but even increasingly popular among practicing cardiologists. The concept seemed so logical and appealing that benefits in surrogate end points, such as blood pressure, proteinuria and endothelial dysfunction, became accepted as a free pass for this combination having cardioprotective and nephroprotective effects. Drs Johannes F.E. Mann and Michael Böhm (pp. 373-377) clearly show that 'it ain't necessarily so' and discuss three randomized controlled trials which ruled out benefits of dual renin-angiotensin blockade on major cardiovascular outcomes and reported substantial adverse effects. Indeed, hyperkalemia, acute renal injury, symptomatic hypotension and syncope were common with dual renin-angiotensin blockade, strongly arguing against such therapy.

Drs Carolina I. Valdiviezo, Lisa Martin and Gurusher S. Panjrath (pp. 378-382) analyze the important question of the target blood pressure during antihypertensive therapy. They thoroughly show that the dictum 'the lower, the better' which was guiding many of us for years is no longer acceptable. Recent data support a target SBP of below $140 \mathrm{mmHg}$ among patients with hypertension or cardiovascular disease, and achievement of this target may, in contrast to some recent guidelines, benefit those older than 60 years of age as well. However, treating to SBPs below $120 \mathrm{mmHg}$ may not result in further benefit. Also, there may be target organ heterogeneity in that the nadir of the J-curve may differ from one cardiovascular outcome to another, that is, the optimal blood pressure for prevention of stroke may be lower than the one for prevention of coronary artery disease.

Finally, we have been taught for decades, mostly by nephrologists, that thiazide diuretics are ineffective antihypertensive agents in moderate-toadvanced renal disease and that in this situation a

Correspondence to Franz H. Messerli, Mount Sinai Health Medical Center, Icahn School of Medicine, Division of Cardiology, New York, NY, USA. Tel: +1 212492 5500; e-mail: messerli.f@gmail.com

Curr Opin Cardiol 2015, 30:359-360

DOI:10.1097/HCO.0000000000000194 
switch to loop diuretics was mandatory. Drs Arjun D. Sinha and Rajiv Agarwal (pp. 366-372) analyze the observational data and small randomized studies including their own suggesting the opposite, namely, that thiazide diuretics can result in meaningful blood pressure reduction in such patients. These data reinforce the need for a randomized trial to demonstrate safety and efficacy of thiazides in advanced chronic kidney disease. The dictum that thiazide diuretics are good antihypertensives but poor diuretics and loop diuretics are poor antihypertensives but good diuretics seems to hold true, at least to some extent in moderate-to-advanced renal disease as well.

\section{Acknowledgements}

None.

Financial support and sponsorship

None.

\section{Conflicts of interest}

There are no conflicts of interest. 